\title{
Catalytic Performance of Al-MCM-48 Molecular Sieves for Isopropylation of Phenol with Isopropyl Acetate
}

\author{
Kandan VENKATACHALAM, Pitchai VISUVAMITHIRAN, Balachandran SUNDARAVEL, \\ Muthiapillai PALANICHAMY, Velayutham MURUGESAN* \\ Department of Chemistry, Anna University, Chennai 600 025, India
}

\begin{abstract}
Al-MCM-48 molecular sieves ( $\mathrm{Si} / \mathrm{Al}$ molar ratios $=25,50,75$, and 100 ) were synthesized hydrothermally using cetyltrimethylammonium bromide as the structure directing template. The orderly arrangement of mesopores was evident from the low angle X-ray diffraction patterns and transmission electron microscopy images. The catalytic performance of the materials was evaluated in the vapor phase isopropylation of phenol with isopropyl acetate. Phenol conversion decreased with the increase in the $\mathrm{Si} / \mathrm{Al}$ ratio of the catalysts. The major reaction product was 4 -isopropyl phenol with $78 \%$ selectivity. The delocalization of phenolic oxygen electron pair over the aromatic ring promoted para-selective alkylation. Such delocalization could be aided by the hydrophilic surface of the molecular sieves. Although an ester was used as the alkylating agent, phenyl isopropyl ether was not formed in the reaction.
\end{abstract}

Key words: Al-MCM-48; alkylation; phenol; isopropyl acetate; 4-isopropyl phenol

CLC number: O643 Document code: A

Received 27 September 2011. Accepted 22 November 2011.

*Corresponding author. Tel: +91-44-22357023/22358645; Fax: +91-44-2220066/22201213; E-mail: v_murugu@hotmail.com

English edition available online at Elsevier ScienceDirect (http://www.sciencedirect.com/science/journal/18722067).

Alkyl phenols are commercially important as they are used as additives in gasoline, lubricants, and consumer products. They are also important intermediates in the synthesis of drugs, agrochemicals, and pharmaceuticals [1]. They are manufactured in industries using mineral acid catalysts such as hydrofluoric acid and sulphuric acid under homogenous condition [2]. As these acid catalysts are hazardous and corrosive in nature, there has been interest to replace them with eco-friendly solid acid catalysts. Phenol is generally susceptible to direct electrophilic attack at ortho and para positions by alkyl cations to form ortho and para alkyl phenols. Meta alkyl phenol is formed by the isomerization of either ortho or para alkyl phenol. Selective alkylation is important for commercial applications, and such selective ortho alkylation has been reported over metal oxide catalysts [3].

Isopropylation of phenol is an important reaction as isopropyl phenols are the precursor for the synthesis of dihydroxy phenol. Isopropyl alcohol is commonly used as alkylating agent. Isopropyl acetate was used as the alkylating agent in the alkylation of phenol in this study due to (i) its easy chemisorption on the Brönsted acid sites to form alkyl cations as the $\mathrm{C}=\mathrm{O}$ bond is highly polar and (ii) release of acetic acid in the reaction that can stabilize the charged structure of phenol. Water also increases the polarity and hydrophilicity of the catalyst surface. Unlike esters, alcohols can not be easily chemisorbed. Alkylation of phenols over solid acid catalysts such as zeolites was reported in the literature [4] but diffusional constrain for both reactants and products prevailed in zeolites. This problem can be circumvented by enlarging the pore size. Mesoporous molecular sieves belonging to the family of M41S [5-10] can avoid this problem. One of the members of this family Al-MCM-41 is attracted for catalytic applications owing to its high thermal stability, large straight pores of uniform size, high surface area, and mild acid sites [5,11]. Closely related to this material is MCM-48 which retains all the features of MCM-41 with additional advantage of crosslinked pores [12] which retard coke formation. It is also expected to give high conversion in catalytic alkylation as it possesses very scattered distribution of acid sites. Jun et al. [13] evaluated the catalytic activity of aluminium incorporated MCM-48 molecular sieves in the Friedel-Crafts alkylation of benzene, toluene, and $m$-xylene with benzyl alcohol. The catalytic activity depends on the preparation methods and the amount of aluminium content. It is also established that post-synthesis incorporation of aluminium is the most effective way to increase the catalytic activity. Dapurkar et al. [14] examined the catalytic activity of Al-MCM-48 catalysts in the tert-butylation of phenol and reported enhanced activity. This enhancement is attributed mainly due to its three-dimensional pore structure. Bhattacharyya et al. [15] reported methylation of phenol over Al-MCM-41. The high acidity favored C-alkylation whereas 
weak acidity favored O-alkylation. Shen et al. [16] reported selective alkylation of phenol with tert-butyl alcohol over [bmim $] \mathrm{PF}_{6}$ and the products were 2,4-IBP, 4-IBP, and 1-IBP. Klerk and Nel [17] reported phenol alkylation with 1-octene over solid phosphoric acid, amorphous silica, aluminates silica, zeolite $\beta$, mordenite, and ZSM-5. Huang et al. [18] reported Al-MCM-48 as a potential hydrotreating catalyst support. The incorporation of aluminium into the framework facilitated enhanced adsorption of light-cycle oil (LCO). Based on the reports of good catalytic performance of Al-MCM-48, isopropylation of phenol using isopropyl acetate (IPA) as alkylating agent was attempted in this reaction. The same reaction was also reported over Al-MCM-41 [19] but exhibited only $47 \%$ conversion with $20.4 \%$ selectivity for 4-isopropyl phenol. This prompted us to study isopropylation of phenol with isopropyl acetate over Al-MCM-48 molecular sieves, as the catalyst has three dimensional constrained pores.

\section{Experimental}

\subsection{Preparation of the catalysts}

Tetraethylorthosilicate (TEOS; Merck) and aluminium hydroxide (Merck) were used as the sources for silicon and aluminium, respectively. Cetyltrimethylammonium bromide (CTAB; Merck) was used as the structure directing agent. Other chemicals such as sodium hydroxide, phenol, and isopropyl acetate were purchased from Merck (India) and used as such without further purification.

The following gel composition was used for the synthesis of Al-MCM-48: TEOS: $x \mathrm{AlO}_{2}: 0.05945 \mathrm{CTAB}: 6 \mathrm{NaOH}$ : $36 \mathrm{H}_{2} \mathrm{O}$. In a typical synthesis of sodium form of Al-MCM48 , TEOS (30.0 g) was added slowly to a solution of CTAB $(31.2 \mathrm{~g})$ dissolved in distilled water (93.6 g). Sodium hydroxide (69 $\mathrm{ml}$ of $1 \mathrm{~mol} / \mathrm{L}$ ) was added to the surfactant solution in order to raise the $\mathrm{pH}$ to 11.6. The gel formed was stirred for $30 \mathrm{~min}$ at room temperature (solution I). The required amount of aluminium hydroxide in $31.2 \mathrm{ml}$ water was stirred for $2 \mathrm{~h}$ at $80^{\circ} \mathrm{C}$ (solution II) and added slowly to solution I. The resulting mixture was then stirred for $1 \mathrm{~h}$ at room temperature. The mixture was transferred to an autoclave $(500 \mathrm{ml})$ and kept in an air-oven at $100{ }^{\circ} \mathrm{C}$ for $72 \mathrm{~h}$. The resultant solid product was washed with distilled water, dried at $100{ }^{\circ} \mathrm{C}$ over night, and calcined at $550{ }^{\circ} \mathrm{C}$ for $1 \mathrm{~h}$ in nitrogen atmosphere followed by $6 \mathrm{~h}$ in air to remove the organic template. The sodium form of Al-MCM-48 was converted to $\mathrm{H}$ form by ion-exchange with $1 \mathrm{~mol} / \mathrm{L}$ ammonium nitrate solution three times followed by calcination at $550{ }^{\circ} \mathrm{C}$ for $6 \mathrm{~h}$ in air.

\subsection{Characterization of the catalysts}

The X-ray diffraction (XRD) patterns of calcined Al-MCM-48 catalysts were recorded on a PANalytical X'Pert Pro X-ray diffractometer using $\mathrm{Cu} K_{\alpha}$ radiation. The low angle diffractograms were recorded in the $2 \theta$ range of $1.0^{\circ}-10^{\circ}$ with a $2 \theta$ step size of $0.01^{\circ}$ and a step time of $10 \mathrm{~s}$ at each point. Surface area measurement was carried out by nitrogen adsorption at $-196{ }^{\circ} \mathrm{C}$ on a ASAP-2010 porosimeter from Micromeritics Corporation (Norcross, GA, USA). Before nitrogen adsorption-desorption measurement, each sample was degassed at $250{ }^{\circ} \mathrm{C}$ for $3 \mathrm{~h}$ under vacuum $\left(10^{-3}\right.$ $\mathrm{Pa})$ in the degas port of the adsorption analyzer. Fourier transform infrared (FT-IR) spectra of the materials were recorded on a FT-IR spectrometer (Nicolet Avatar 360) using $\mathrm{KBr}$ pellet technique. About $15 \mathrm{mg}$ of the sample was pressed (under a pressure of 2 tons $/ \mathrm{cm}^{2}$ ) into a self-supported wafer of $13 \mathrm{~mm}$ diameter. This pellet was used to record the infrared spectrum in the range $4000-400 \mathrm{~cm}^{-1}$. Thermogravimetric analysis (TGA) of as-synthesized Al-MCM-48 was carried out using thermogravimetric analyzer (Perkin Elmer Diamond series). The sample was heated in a flow of nitrogen at a heating rate of $10{ }^{\circ} \mathrm{C} / \mathrm{min}$ in the temperature range of $50-700{ }^{\circ} \mathrm{C}$.

Scanning electron microscopic (SEM) images were recorded using a SEM (Hitachi S-4500LV) instrument. Transmission electron microscopic (TEM) images were recorded using a high resolution TEM instrument (JEOL 3010 electron microscope) operated at $300 \mathrm{kV}$. The powder sample was grounded in an agate mortar and dispersed in acetone in an ultrasonic bath for several minutes. A few drops were then deposited on a 200 mesh copper grid covered with a holey carbon film. Mesostructure of the solid was imaged directly by conventional method, and also using CRISP computer program.

\subsection{Catalytic studies}

Alkylation of phenol with isopropyl acetate was carried out in a tubular fixed-bed, vertical flow-type glass reactor of $40 \mathrm{~cm}$ length and $2 \mathrm{~cm}$ internal diameter. The reaction was carried out at atmospheric pressure in the temperature range $200-300{ }^{\circ} \mathrm{C}$. About $0.5 \mathrm{~g}$ of the catalyst was placed in the middle of the reactor and supported on either side with a thin layer of quartz wool and ceramic beads. The reactor was heated to the requisite temperature with the help of a tubular furnace controlled by a digital temperature controller cum indicator. The catalyst was activated in air at $500{ }^{\circ} \mathrm{C}$ for $5 \mathrm{~h}$ prior to each catalytic reaction. A mixture of phenol and isopropyl acetate was fed into the reactor using a syringe infusion pump at a predetermined flow rate. The bottom of the reactor was connected to a coiled condenser and a receiver to collect the products. The products obtained in the first $10 \mathrm{~min}$ were discarded, and that collected thereafter 
were analyzed by a gas chromatograph (Shimadzu model GC 17A) using DB-5 capillary column equipped with a flame ionization detector and $\mathrm{N}_{2}$ as the carrier gas at a flow rate of $20 \mathrm{ml} / \mathrm{min}$. The identification of the products was also made using a gas chromatograph (Perkin Elmer auto-system XL elite series PE-5 capillary column) equipped with a mass spectrometer (Turbo, EI $70 \mathrm{eV}$ ) using helium as the carrier gas at a flow rate of $1 \mathrm{ml} / \mathrm{min}$.

\section{Results and discussion}

\subsection{XRD}

The XRD patterns of the calcined Al-MCM-48 ( $\mathrm{Si} / \mathrm{Al}=$ 25, 50, 75, and 100) are shown in Fig. 1. The peak due to (211) was present in all the patterns but the peak due to (220) was not well resolved. Although Zhao et al. [20] reported well resolved patterns for MCM-48, such patterns were not observed in our study. The peaks due to (420) and (332) overlapped in all the patterns without enough resolution. Similar XRD patterns were also reported by Huang et al. [21]. The intensity of the peak due to (211) plane increased with increase of $\mathrm{Si} / \mathrm{Al}$ ratio of the materials. Though TEM results revealed orderly arrangement of pores as discussed below, minute variation in wall thickness might exist. Such variation might be the cause for the absence of resolved peaks due to (420) and (332).

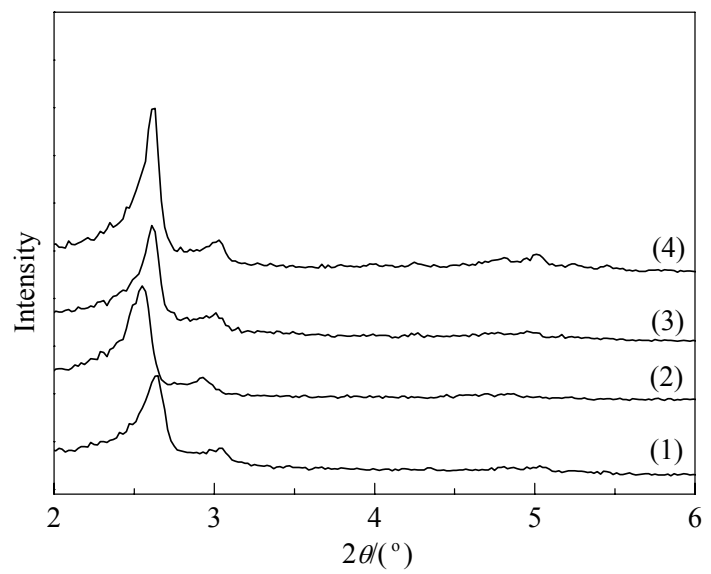

Fig. 1. XRD patterns of the calcined Al-MCM-48(25) (1), Al-MCM48(50) (2), Al-MCM-48(75) (3), and Al-MCM-48(100) (4).

\section{$2.2 \quad \mathrm{~N}_{2}$ adsorption-desorption}

The nitrogen adsorption-desorption isotherms of Al-MCM-48 $(\mathrm{Si} / \mathrm{Al}=25,50,75$, and 100$)$ are shown in Fig. 2. All the isotherms exhibit the characteristic type IV adsorption [22]. There are two hysteresis loops in all the isotherms, the first one occurs at low $p / p_{0}$ ratio due to condensation of nitrogen in the capillary, and the second one occurs

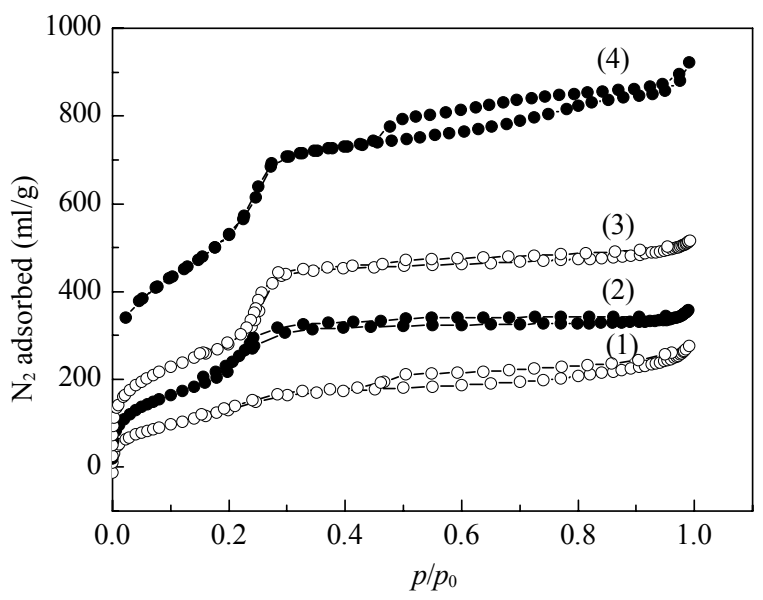

Fig. 2. $\mathrm{N}_{2}$ adsorption-desorption isotherms of Al-MCM-48(25) (1), Al-MCM-48(50) (2), Al-MCM-48(75) (3), and Al-MCM-48(100) (4).

at high $p / p_{0}$ due to condensation in the voids formed between the particles [23]. Hence the size of the void may be larger in dimension than the pore size. Comparison of the height due to pore condensation illustrates that the pore volume of Al-MCM-48(100) is higher than that of Al-MCM-48(75), Al-MCM-48(50), and Al-MCM-48(25). The smooth increase in condensation inside the pores illustrates uniform pore size distribution.

The BET surface area, pore volume, and pore size computed from the nitrogen adsorption isotherms are presented in Table 1. The surface area, pore volume, and pore size decreased with increase in the aluminium content of the molecular sieves. When silicon is substituted by aluminium, these parameters are expected to increase because of higher bond angles of aluminium tetrahedra than silicon tetrahedra. But the values are not in accordance with expected increase. When aluminium is planted in the framework of MCM-48, the framework carries negative charge. With this negative charge the framework can move close to the surface of the micelle rods that carries positive charge. Such a close approach is suggested to be main cause for the decrease in the values of these parameters with increase of aluminium content.

Table 1 Physico-chemical characteristics of the catalysts

\begin{tabular}{lccc}
\hline Catalyst & $\begin{array}{c}\text { Specific surface } \\
\text { area }\left(\mathrm{m}^{2} / \mathrm{g}\right)\end{array}$ & $\begin{array}{c}\text { Pore volume } \\
\left(\mathrm{cm}^{3} / \mathrm{g}\right)\end{array}$ & $\begin{array}{c}\text { Pore size } \\
(\mathrm{nm})\end{array}$ \\
\hline Al-MCM-48(25) & 1247 & 0.54 & 2.12 \\
Al-MCM-48(50) & 1293 & 0.62 & 2.20 \\
Al-MCM-48(75) & 1317 & 0.71 & 2.23 \\
Al-MCM-48(100) & 1342 & 0.76 & 2.27 \\
\hline
\end{tabular}

\section{$2.3 \quad \mathrm{NH}_{3}$-TPD}

The results of $\mathrm{NH}_{3}-\mathrm{TPD}$, derived for all the catalysts, are 
depicted in Fig. 3. The maxima were found between 175 and $350{ }^{\circ} \mathrm{C}$ in all the cases. Hence weak and medium acid sites are predicted in all the catalysts. The maxima as well as the area decreased with increase of $\mathrm{Si} / \mathrm{Al}$ ratio. Similar results were reported earlier for mesoporous materials $[24,25]$. The maxima are shifted to lower temperature with the increase in $\mathrm{Si} / \mathrm{Al}$ ratio. So the effect of $\mathrm{Si} / \mathrm{Al}$ ratio on the acid strength predicted for zeolites may not be extended to mesoporous materials. The amorphous nature of the wall of the mesoporous materials might be the reason for this trend. The walls are uniatomic in zeolite and so the acidity of protons is strongly influenced by $\mathrm{Si} / \mathrm{Al}$ ratio. However, the walls are of multiatom thickness in mesoporous materials and so the $\mathrm{Si} / \mathrm{Al}$ ratio can not directly influence the acidity.

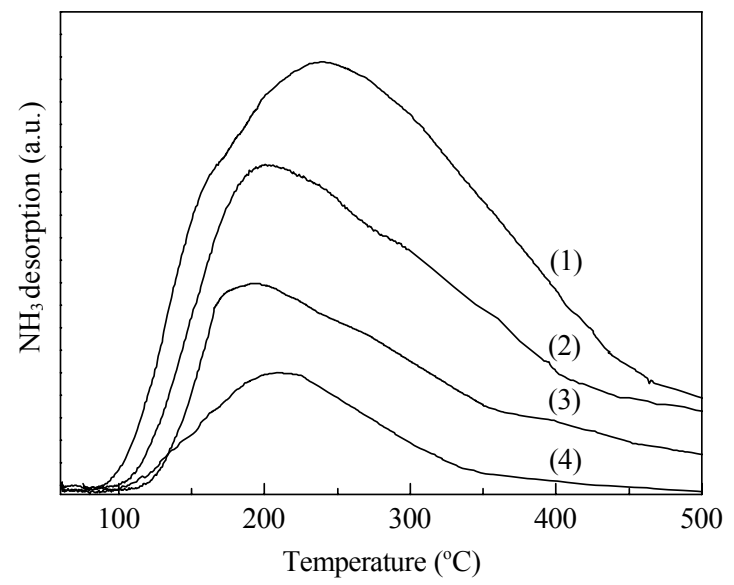

Fig. 3. $\mathrm{NH}_{3}-\mathrm{TPD}$ profiles of Al-MCM-48(25) (1), Al-MCM-48(50) (2), Al-MCM-48(75) (3), and Al-MCM-48(100) (4).

\subsection{FT-IR}

The FT-IR spectra of as-synthesized Al-MCM-48 (Si/Al $=25,50,75$, and 100) are shown in Fig. 4(a). All the spectra showed similar features. The intense broad envelope between 3000 and $3740 \mathrm{~cm}^{-1}$ was due to $-\mathrm{OH}$ stretching vibration of water. The peak due to stretching vibration of defective $\mathrm{Si}-\mathrm{O}-\mathrm{H}$ groups merged with the envelope of $-\mathrm{OH}$ stretching vibration of water. The peak around $1630 \mathrm{~cm}^{-1}$ was assigned to the bending mode of water. The symmetric and asymmetric vibrations of $-\mathrm{CH}_{2}$ groups of the structure directing template were located just below $3000 \mathrm{~cm}^{-1}$. The bending modes of $-\mathrm{CH}_{2}$ groups exhibited their peaks between 1500 and $1300 \mathrm{~cm}^{-1}$. The asymmetric stretching vibrations of $\mathrm{Si}-\mathrm{O}-\mathrm{Si}$ and $\mathrm{Si}-\mathrm{O}-\mathrm{Al}$ groups were intense and broad, and appeared between 1000 and $1300 \mathrm{~cm}^{-1}$. Their defective $\mathrm{Si}-\mathrm{O}-\mathrm{H}$ vibration occurred just below $1000 \mathrm{~cm}^{-1}$. The symmetric stretching vibration of $\mathrm{Si}-\mathrm{O}-\mathrm{Si}$ bonds occurred at about $760 \mathrm{~cm}^{-1}$ and their bending mode at 490 $\mathrm{cm}^{-1}$. Similar spectra were also reported by Dapurkar et al. [14]. The asymmetric stretching vibration resulted a broad

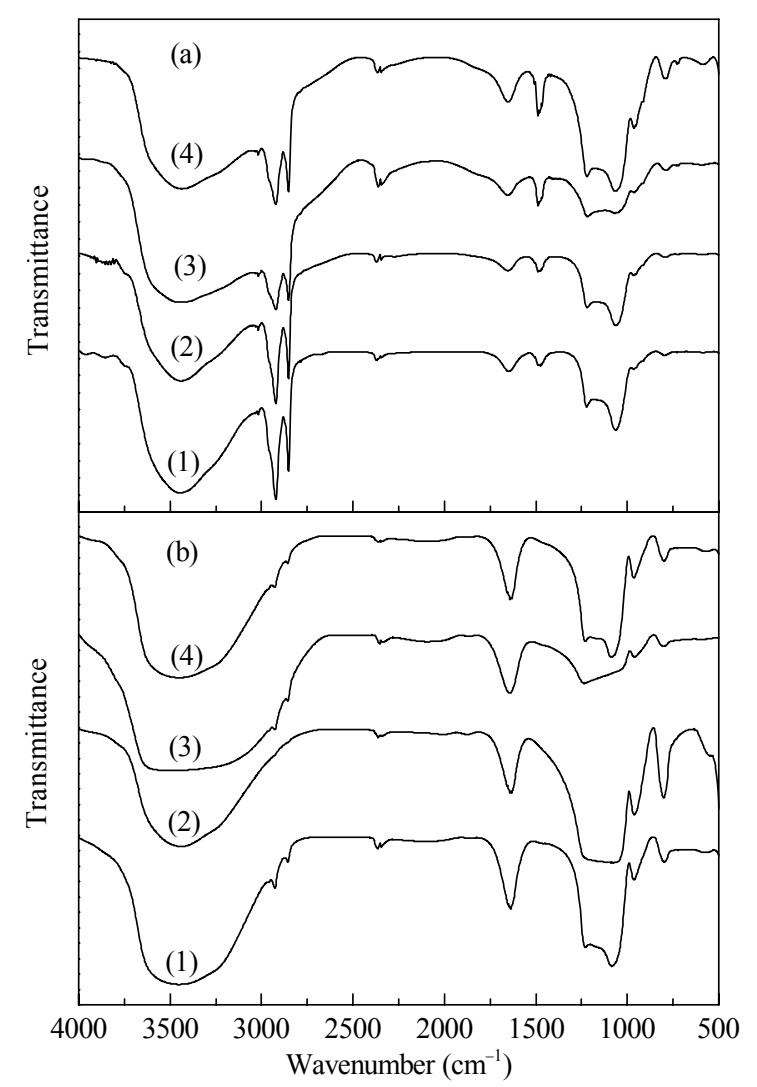

Fig. 4. FT-IR spectra of as-synthesized (a) and calcined (b) Al-MCM-48(25) (1), Al-MCM-48(50) (2), Al-MCM-48(75) (3), and Al-MCM-48(100) (4).

peak between 1250 and $1000 \mathrm{~cm}^{-1}$. The $\mathrm{Si}-\mathrm{OH}$ vibration occurred just below $1000 \mathrm{~cm}^{-1}$. The $\mathrm{Si}-\mathrm{O}-\mathrm{Si}$ symmetric starching vibration and bending mode also occurred below $1000 \mathrm{~cm}^{-1}$.

The FT-IR spectra of calcined Al-MCM-48 ( $\mathrm{Si} / \mathrm{Al}=25$, 50, 75, and 100) are shown in Fig. 4(b). All the spectra exhibited nearly similar features as that of the as-synthesized materials except the peaks due to template vibrations. The intensity of the peak corresponding to the stretching and bending modes of water were clearly seen. Hence, the hydrophilic property of the calcined materials is evident from these spectra.

\subsection{TGA}

The thermogram of Al-MCM-48(25) is shown in Fig. 5. The mass loss below $175{ }^{\circ} \mathrm{C}$ was gradual but above that temperature there was a major mass loss extending up to $300{ }^{\circ} \mathrm{C}$. The former mass loss was due to desorption of water and the later due to desorption and degradation of template. The minute mass loss between 300 and $450{ }^{\circ} \mathrm{C}$ was attributed to the condensation of defective hydroxyl groups. These results are in accordance with those reported by $\mathrm{Xu}$ et al. [26]. 


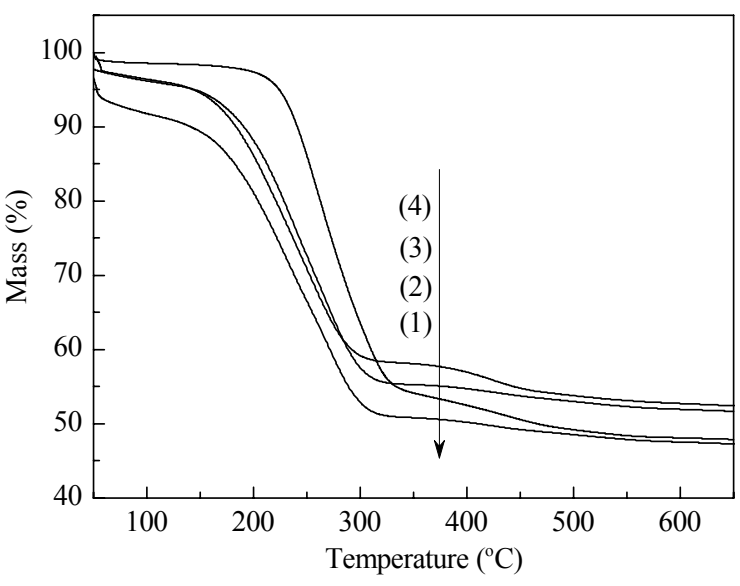

Fig. 5. TGA curves of as-synthesized Al-MCM-48(25) (1), Al-MCM-48(50) (2), Al-MCM-48(75) (3), and Al-MCM-48(100) (4).

\subsection{SEM and TEM}

The SEM images of Al-MCM-48 are shown in Fig. 6. It exhibited particles of spherical morphologies. Such morphologies were also reported in the literature [22]. Though the particles were of different sizes, other phases were absent.

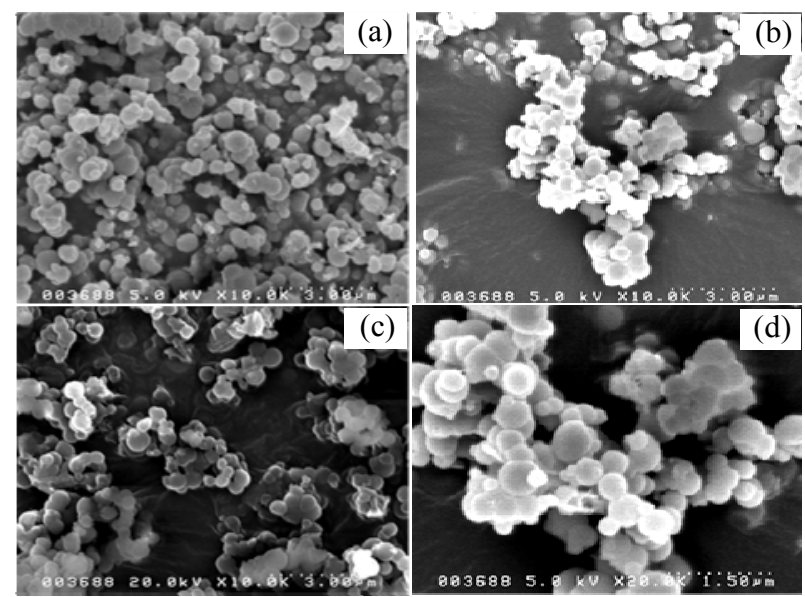

Fig. 6. SEM images of Al-MCM-48(25) (a), Al-MCM-48(50) (b), Al-MCM-48(75) (c), and Al-MCM-48(100) (d).

The TEM images of Al-MCM-48(25) is shown in Fig. 7. All the pictures showed that the pores are arranged in an orderly arrangement, illustrating textural uniformity. Similar images were already reported in the literature [15].

\subsection{Catalytic studies}

The catalytic activity of Al-MCM-48 (Si/Al ratios 25, 50, 75 , and 100) was evaluated in the vapor phase isopropylation of phenol with isopropyl acetate (IPA) at 200, 225, 250, 275 , and $300{ }^{\circ} \mathrm{C}$. The feed ratio (phenol:IPA) and WHSV

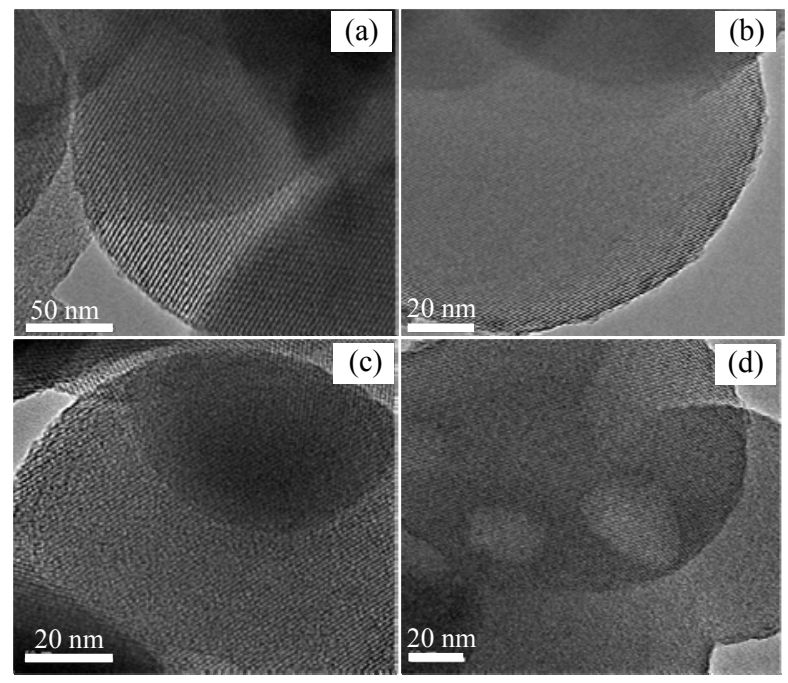

Fig. 7. TEM images of Al-MCM-48(25) (a), Al-MCM-48(50) (b), Al-MCM-48(75) (c), and Al-MCM-48(100) (d).

were $1: 3$ and $5.32 \mathrm{~h}^{-1}$, respectively. The major products were found to be 2-isopropyl phenol (2-IPP), 4-isopropyl phenol (4-IPP), and 3-isopropyl phenol (3-IPP), in addition to small amount of isomers of diisopropyl phenols. The amount of acylated products formed in the reaction was very much low in the entire temperature range. The results of phenol conversion and products selectivity are presented in Table 2. The phenol conversion increased from 200 to $250{ }^{\circ} \mathrm{C}$ but thereafter it decreased gradually due to coke formation [19]. The phenol conversion was $62 \%$ at $200{ }^{\circ} \mathrm{C}$ whereas it was $78 \%$ at $225^{\circ} \mathrm{C}$. This large difference in conversion is attributed to enhanced activation of reactants and three-dimensional cross linked pores which could avoid crowding of molecules at any single active sites. The selectivity for 4-IPP was higher than all others, and the order of selectivity was 4-IPP > 3-IPP > 2-IPP. The selectivity of all other products including isomers of diisopropylated phenols and traces of acylated products are presented in the last column of Table 2.

The selectivity for 2-IPP decreased with increase in temperature from 200 to $275{ }^{\circ} \mathrm{C}$ but increased at $300{ }^{\circ} \mathrm{C}$. The decrease was attributed to isomerization of 2-IPP to 3-IPP which was supported by the increase in the selectivity for 3-IPP with increase in temperature. The decrease in the selectivity for 3-IPP with increase in temperature may not be due to suppression of isomerization but due to its further alkylation to diisopropylated products particularly 2,5-diisopropylphenols. The formation of the principal product, 4-IPP, can be accounted as follows. There is resonance delocalization of oxygen lone pair electrons on the benzene ring of phenol. This delocalization results in the formation of partial positive charge on the oxygen. When phenol approaches the isopropyl cation resting as the charge 
Table 2 Effect of temperature on phenol conversion and product selectivity

\begin{tabular}{|c|c|c|c|c|c|c|}
\hline \multirow{2}{*}{ Catalyst } & \multirow{2}{*}{$T /{ }^{\circ} \mathrm{C}$} & \multirow{2}{*}{$\begin{array}{l}\text { Conver- } \\
\text { sion }(\%)\end{array}$} & \multicolumn{4}{|c|}{ Selectivity (\%) } \\
\hline & & & 2-IPP & 4-IPP & 3-IPP & Iso $^{*}$ \\
\hline \multirow[t]{5}{*}{ Al-MCM-48(25) } & 200 & 62 & 9 & 60 & 23 & 8 \\
\hline & 225 & 78 & 6 & 66 & 17 & 11 \\
\hline & 250 & 89 & 3 & 72 & 13 & 12 \\
\hline & 275 & 83 & 4 & 69 & 10 & 17 \\
\hline & 300 & 80 & 7 & 65 & 9 & 19 \\
\hline \multirow[t]{5}{*}{ Al-MCM-48(50) } & 200 & 59 & 14 & 52 & 25 & 9 \\
\hline & 225 & 63 & 10 & 56 & 21 & 13 \\
\hline & 250 & 71 & 7 & 62 & 17 & 14 \\
\hline & 275 & 68 & 9 & 59 & 14 & 18 \\
\hline & 300 & 65 & 11 & 56 & 13 & 20 \\
\hline \multirow[t]{5}{*}{ Al-MCM-48(75) } & 200 & 56 & 16 & 43 & 29 & 12 \\
\hline & 225 & 61 & 13 & 45 & 25 & 17 \\
\hline & 250 & 67 & 8 & 53 & 21 & 18 \\
\hline & 275 & 63 & 11 & 49 & 19 & 21 \\
\hline & 300 & 61 & 12 & 46 & 17 & 23 \\
\hline \multirow[t]{5}{*}{ Al-MCM-48(100) } & 200 & 55 & 17 & 40 & 30 & 13 \\
\hline & 225 & 59 & 14 & 42 & 28 & 16 \\
\hline & 250 & 61 & 10 & 49 & 20 & 21 \\
\hline & 275 & 58 & 13 & 44 & 21 & 22 \\
\hline & 300 & 57 & 14 & 41 & 20 & 25 \\
\hline
\end{tabular}

Reaction conditions: catalyst amount $0.5 \mathrm{~g}$, phenol:isopropyl acetate feed ratio $1: 3$, time $1 \mathrm{~h}$, WHSV $=5.32 \mathrm{~h}^{-1}$.

"Isomers of diisopropyl phenols and acetic acid.

compensating cation on the channel surface, the ortho positions of phenol may not be suitable for electrophilic attack by isopropyl cation, as the adjacent phenolic $-\mathrm{OH}$ group with its positive charge may repel it. Because the para carbon is comparatively free of such repulsion, the isopropyl cation freely attacks the para-carbon to form 4-IPP. Hence, this product is formed with higher selectivity than others at all temperatures. The selectivity increased from 200 to 275 ${ }^{\circ} \mathrm{C}$ but decreased thereafter. The decrease is due to the conversion of 4-IPP to diisopropylated products. This is presumed to be true as dialkylation is a high activation energy demanding reaction [27].

The selectivity for 3-IPP should increase with increase in temperature but it showed a reverse formed. This product is thermodynamically more stable than para and ortho products, and it is formed mainly by the isomerization of para and ortho products. Since isomerization is high activation energy demanding, selectivity for meta product should increase with increase in temperature. Contrary to the expectation, the selectivity for 3-IPP decreased due to the formation of coke which suppressed isomerization as well as its conversion to di-isopropylated phenols. Based on the discussion the following scheme is proposed to account for the products distribution. Isopropyl acetate is chemisorbed on the Brönsted acid site of the catalyst to form isopropyl cation, which in turn reacts with phenol to form ortho and para-isopropyl phenols. These products undergo further isomerization yielding 3-IPP as shown in the reaction Scheme 1. All the mono-isopropyl phenol isomers subsequently undergo further isopropylation to form di-isopropylated phenols as shown in the same reaction scheme.

The conversion of phenol and selectivity for products obtained over Al-MCM-48(50) are shown in Table 2. The conversion was slightly lower than Al-MCM-48(25) due to decrease in acidity as shown by $\mathrm{NH}_{3}-\mathrm{TPD}$. Though aluminium content in Al-MCM-48(50) was 50\% less than Al-MCM-48(25), the conversion was not lowered by $50 \%$. It could be speculated that Brönsted acid sites in these catalysts may be very much dispersed compared to Al-MCM-48(25). The same reaction was also studied over Al-MCM-41 by Savitha et al. [19]. They reported only $47 \%$ phenol conversion at $300{ }^{\circ} \mathrm{C}$ under the same conditions. However, Al-MCM-48 exhibited 65\% phenol conversion. The high conversion over Al-MCM-48 is attributed to high dispersion of acid sites in this catalyst. The selectivity for 4-IPP over Al-MCM-48(50) was also slightly lower than Al-MCM-48(25). Comparison of the selectivity for other products showed an increase in the selectivity for 2-IPP and 3-IPP. In addition, the selectivity for diisopropyl phenols also increased slightly. All these observations could be accounted by the influence of Lewis acid sites. These Lewis acid sites were formed during calcination. The results of phenol conversion and product selectivity over Al-MCM-48(75) and Al-MCM-48(100) also revealed nearly similar trend as that of Al-MCM-48(50). The catalytic activity of Si-MCM-48 was tested at $200{ }^{\circ} \mathrm{C}$ with feed ratio 1:3 and WHSV $5.32 \mathrm{~h}^{-1}$. There was no significant conversion $(<1 \%)$ indicating that the reaction was evidently catalyzed by Brönsted acid sites.

The effect of feed ratio on phenol conversion and products selectivity over Al-MCM-48(25) was studied with 1:1, $1: 2,1: 3,1: 4$, and 1:5 (phenol:isopropyl acetate) and WHSV $5.32 \mathrm{~h}^{-1}$ at $250{ }^{\circ} \mathrm{C}$. The results of phenol conversion and products selectivity are presented in Table 3 . The conversion increased from $1: 1$ to $1: 3$ feed ratio but decreased thereafter. The decrease in conversion at 1:4 and 1:5 was due to dilution of phenol by the presence of excess IPA. Such observations were already reported in the literature [19]. The increase in conversion from $1: 1$ to $1: 3$ is due to enhanced availability of isopropyl cation for alkylation. The selectivity for 2-IPP was not more than $10 \%$ over all the catalysts. The selectivity for 4-IPP increased from 1:1 to 1:3 but decreased at 1:4 and 1:5. The decrease was due to isomerization of 4-IPP to 3-IPP. Since isopropylation of phenol is suppressed due to dilution by excess IPA, the preformed 4-IPP can very well undergo isomerization either on the 


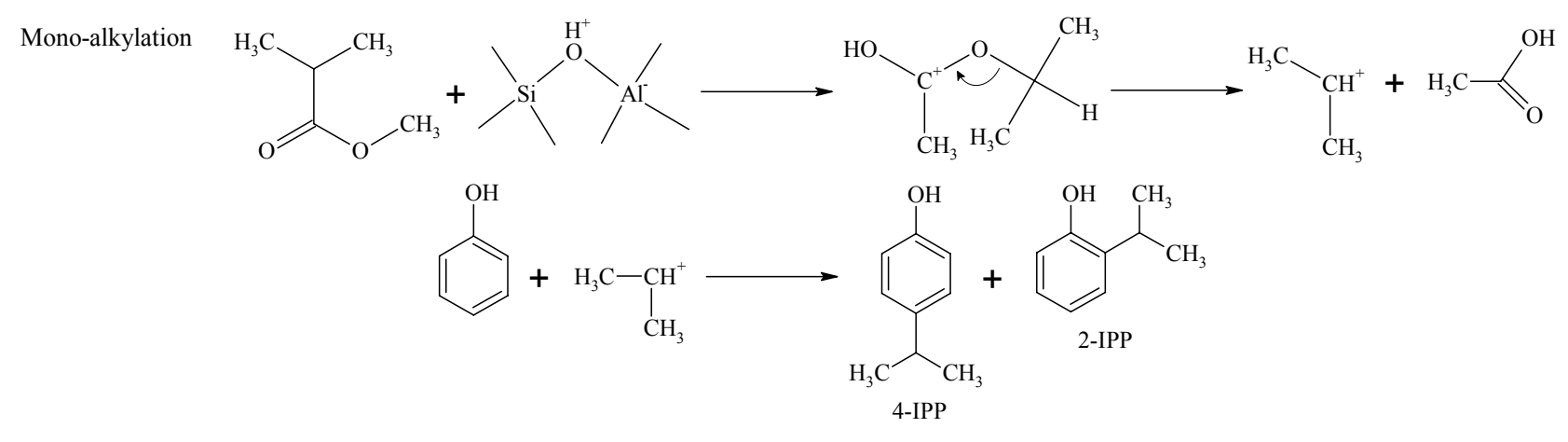

Di-alkylation<smiles>CC(C)c1ccc(O)cc1</smiles>

2,4-Diisopropylphenol

Isomerization<smiles>CC(C)c1ccccc1O</smiles><smiles>C[Si](C)(C)O[I+](C)(C)C</smiles><smiles>CCCCCCCC</smiles>

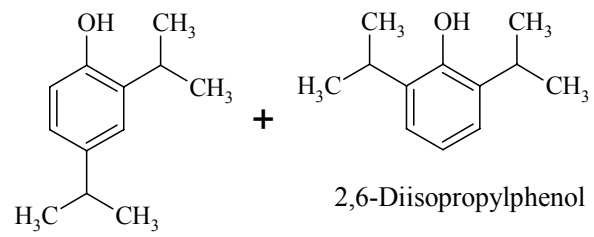

\section{2,4-Diisopropylphenol}

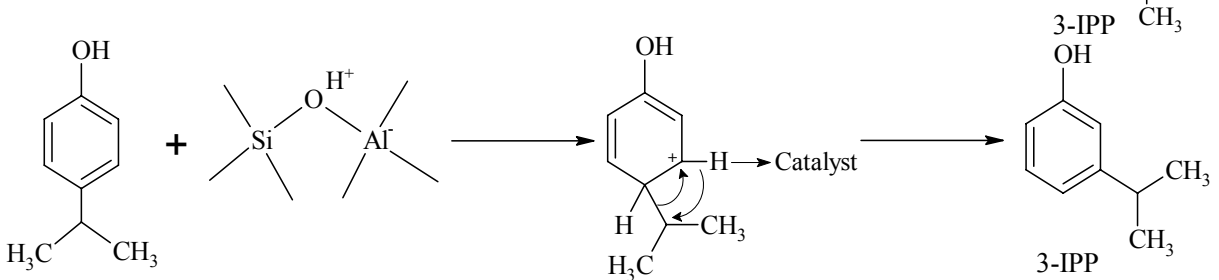

Scheme 1. The proposed mechanism for mono-alkylation, di-alkylation, and isomerization.

Table 3 Effect of phenol:isopropyl acetate feed ratio on phenol conversion and product selectivity over Al-MCM-48(25)

\begin{tabular}{cccccc}
\hline \multirow{2}{*}{$\begin{array}{c}\text { Feed } \\
\text { ratio }\end{array}$} & $\begin{array}{c}\text { Conversion } \\
(\%)\end{array}$ & \multicolumn{4}{c}{ Selectivity (\%) } \\
\cline { 3 - 6 } $1: 1$ & 70 & 10 & 73 & 13 & 4 \\
$1: 2$ & 83 & 10 & 75 & 9 & 6 \\
$1: 3$ & 92 & 5 & 78 & 10 & 7 \\
$1: 4$ & 89 & 4 & 72 & 18 & 6 \\
$1: 5$ & 87 & 4 & 70 & 21 & 5 \\
$2: 1$ & 52 & 3 & 63 & 25 & 9 \\
$3: 1$ & 40 & 2 & 62 & 29 & 7 \\
\hline
\end{tabular}

Reaction conditions: catalyst amount $0.5 \mathrm{~g}$, time $1 \mathrm{~h}$, temperature 250 ${ }^{\circ} \mathrm{C}$.

same sites on which it is formed or other sites that could be available in the immediate vicinity. Hence it is concluded that the feed ratio of 1:3 was better than all others as it gave high phenol conversion and high selectivity for 4-IPP. The reaction was also studied with feed ratio $2: 1$ and 3:1 but the conversion significantly decrease, as the alkyl cation for alkylation was very much reduced for the selectivity of 4-IPP. But the isomerisation to 3-IPP was enhanced. The enhancement of isomerisation of 4-IPP is due to reduced isopropyl acetate content.

The effect of WHSV on phenol conversion and products selectivity was examined over AlMCM-48(25) with the feed ratio $1: 3$ at $250{ }^{\circ} \mathrm{C}$. The results of phenol conversion and product selectivity are given in Table 4 . The conversion showed a non-linear response to space velocity. The conversion increased when the WHSV 2.62 to $5.32 \mathrm{~h}^{-1}$. Though at $5.32 \mathrm{~h}^{-1}$ contact time decreased, the concentration of isopropyl acetate around the active sites increased. Hence the 
Table 4 Effect of WHSV on phenol conversion and product selectivity over Al-MCM-48(25)

\begin{tabular}{cccccc}
\hline WHSV & Conversion & \multicolumn{4}{c}{ Selectivity (\%) } \\
\cline { 3 - 6 }$\left(\mathrm{h}^{-1}\right)$ & $(\%)$ & 2-IPP & 4-IPP & 3-IPP & Iso \\
\hline 2.62 & 71 & 4 & 72 & 18 & 6 \\
5.32 & 92 & 5 & 78 & 10 & 7 \\
9.19 & 89 & 25 & 70 & 7 & 6 \\
12.87 & 78 & 27 & 63 & 6 & 4 \\
\hline
\end{tabular}

Reaction conditions: catalyst amount $0.5 \mathrm{~g}$, time $1 \mathrm{~h}$, temperature 250 ${ }^{\circ} \mathrm{C}$, feed ratio $1: 3$.

conversion of phenol increased. The concentration of isopropyl acetate could increase around the active sites at 9.19 and $12.87 \mathrm{~h}^{-1}$, but the contact time was reduced and hence the conversion of phenol decreased. But the selectivity for 4-IPP was higher at WHSV of $5.32 \mathrm{~h}^{-1}$ than others. The selectivity for 2-IPP increased with increase in WHSV since its isomerization to 3-IPP could be suppressed with increase in WHSV. In addition, its consecutive alkylation to dialkylated phenols may also be suppressed with increase WHSV. Coke formation may not be the main cause as there was high phenol conversion.

The effect of time on stream on phenol conversion and products selectivity was studied over Al-MCM-48(25) at $250{ }^{\circ} \mathrm{C}$ with the feed ratio $1: 3$ and WHSV $5.32 \mathrm{~h}^{-1}$. The results are shown in Table 5. The phenol conversion decreased with increase in time on stream, but the decrease was not high at the end of first and second hour. Hence, the rate of coke formation in the first two hours of time on stream may be low. Even at the end of three hours, the conversion was found to be $89 \%$. The conversion decreased rapidly only after three hours of time on stream, which is attributed to substantial formation of coke. Deactivation of Al-MCM-48 due to coke formation was reported to be slow because of its interconnected three dimensional mesopore [12,14,28,29]. The selectivity for 2-IPP increased with increase in time on stream. This is due to coke formation at latter stage of time on stream. The formation of coke could suppress isomerization of 2-IPP or its consecutive alkylation to diisopropyl phenol isomers. The selectivity for 4-IPP decreased in the latter stage of time on stream. This study

Table 5 Effect of time on stream on phenol conversion and product selectivity over Al-MCM-48(25)

\begin{tabular}{cccccc}
\hline \multirow{2}{*}{$\begin{array}{c}\text { Time } \\
(\mathrm{h})\end{array}$} & \multirow{2}{*}{\begin{tabular}{c} 
Conversion \\
\cline { 3 - 6 }
\end{tabular}} & \multicolumn{5}{c}{ Selectivity (\%) } \\
\cline { 3 - 6 } 1 & 92 & 5 & 78 & 10 & 7 \\
2 & 92 & 13 & 72 & 9 & 6 \\
3 & 89 & 19 & 70 & 7 & 4 \\
4 & 83 & 24 & 69 & 4 & 3 \\
5 & 79 & 30 & 64 & 3 & 3 \\
\hline
\end{tabular}

Reaction conditions: catalyst amount $0.5 \mathrm{~g}$, temperature $250{ }^{\circ} \mathrm{C}$, feed ratio $1: 3$. thus illustrates the requirement of frequent activation of catalyst after conducting the reaction for at least two hours in order to exhibit high conversion and high selectivity for 4-IPP.

\section{Conclusions}

Al-MCM-48 molecular sieves were synthesized hydrothermally using cetyltrimethylammonium bromide as the structure directing template. The pore size of the materials was in the range of $2.12-2.27 \mathrm{~nm}$ and the surface area $1247-1342 \mathrm{~m}^{2} / \mathrm{g}$. Isopropylation of phenol with IPA was carried out over all the catalysts. More than $90 \%$ phenol conversion was obtained and the products were found to be 2-IPP, 4-IPP, and 3-IPP. The order of the catalyst activity was Al-MCM-48(25) > Al-MCM-48(50) > Al-MCM-48(75) $>$ Al-MCM-48(100). Like other mesoporous molecular sieves, Al-MCM-48 is also a convenient catalyst for alkylation of aromatics. Its cross-linked pores are important as it can suppress coke formation and promote high dispersion of active sites. Like other mesoporous molecular sieves, it also carries only weak acid sites and hence isomerization of alkyl aromatics was low.

\section{Acknowledgements}

The authors gratefully acknowledge the financial support from the Department of Science and Technology (Sanction No. SR/S1/PC-10/2009, Dated 28.10.2009). The authors also acknowledge University Grants Commission (UGC), New Delhi for providing funds under special assistance DRS programme and Department of Science and Technology, New Delhi, under FIST programme for equipment facilities in the Department.

\section{References}

1 Knop A, Pilato L A. Phenolic Resins Chemistry. Berlin: Springer, 1985

2 Cao J M, He N Y, Li C, Dong J L, Xu Q H. Stud Surf Sci Catal, 1998, 117: 461

3 Kotanigawa T. Bull Chem Soc Jpn, 1974, 47: 950

4 Kresge C T, Leonowicz M E, Roth W J, Vartuli J C, Beck J S. Nature, 1992, 359: 710

5 Beck J S, Vartuli J C, Roth W J, Leonowicz M E, Kresge C T, Schmitt K D, Chu C T W, Olson D H, Sheppard E W, McCullen S B, Higgins J B, Schlenker J L. J Am Chem Soc, 1992, 114: 10834

6 Ariga K, Vinu A, Hill J P, Mori T. Coord Chem Rev, 2007, 251: 2562

7 Ariga K, Vinu A, Miyahara M, Hill J P, Mori T. J Am Chem Soc, 2007, 129: 11022

8 Ji Q M, Miyahara M. Hill J P, Acharya S, Vinu A, Yoon S B, 
Yu J-S, Sakamoto K, Ariga K. J Am Chem Soc, 2008, 130: 2376

9 Ji Q M, Yoon S B, Hill J P, Vinu A, Yu J-S, Ariga K. J Am Chem Soc, 2009, 131: 4220

10 Ji Q M, Acharya S, Hill J P, Vinu A, Yoon S B, Yu J-S, Sakamoto K, Ariga K. Adv Funct Mater, 2009, 19: 1792

11 Behrens P, Stucky G D. Angew Chem, Int Ed, 1993, 32: 696

12 Krithiga T, Vinu A, Ariga K, Arabindoo B, Palanichamy M, Murugesan V. J Mol Catal A, 2005, 237: 238

13 Jun S, Ryoo R. J Catal, 2000, 195: 237

14 Dapurkar S E, Selvam P. Appl Catal A, 2003, 254: 239

15 Bhattacharyya K G, Talukdar A K, Das P, Sivasanker S. J Mol Catal A, 2003, 197: 255

16 Shen H-Y, Judeh Z M A, Ching C B. Tetrahedron Lett, 2003, 44: 981

17 de Klerk A, Nel R J J. Ind Eng Chem Res, 2007, 46: 7066

18 Huang L, Huang Q L, Xiao H N, Eic M. Microporous Mesoporous Mater, 2008, 114: 121

19 Savidha R, Pandurangan A, Palaichamy M, Murugesan V.
Catal Lett, 2003, 91: 49

20 Zhao W, Hao Z P, Hu C. Mater Res Bull, 2005, 40: 1775

21 Huang L, Huang Q L, Xiao H N, Eic M. Microporous Mesoporous Mater, 2008, 111: 404

22 Wang L Z, Shao Y F, Zhang J L. Mater Lett, 2005, 59: 3604

23 Russo P A, Manuela M, Carrott L R, Carrott P J M. Colloids Surf A, 2007, 310: 9

24 Ko A-N, Hung C-C, Chen C-W, Ouyang K-H. Catal Lett, 2001, 71: 219

25 Park H J, Yim J-H, Jeon J-K, Kim J M, Yoo K-S, Park Y-K. J Phys Chem Solids, 2008, 69: 1125

26 Xu J, Luan Z H, Hartmann M, Kevan L. Chem Mater, 1999, 11: 2928

27 Colon G, Ferino I, Rombi E, Selli E, Forni L, Magnoux P, Guisnet M. Appl Catal A, 1998, 168: 81

28 Kosslick H, Lischke G, Landmesser H, Partitz B, Storek W, Fricke R. J Catal, 1998, 176: 102

$29 \mathrm{Pu} \mathrm{S}$ B, Kim J B, Seno M, Inui T. Microporous Mesoporous Mater, 1997, 10: 25 